\title{
Unraveling the Gymnastics of Reverse Transcription Through Single Molecule Spectroscopy
}

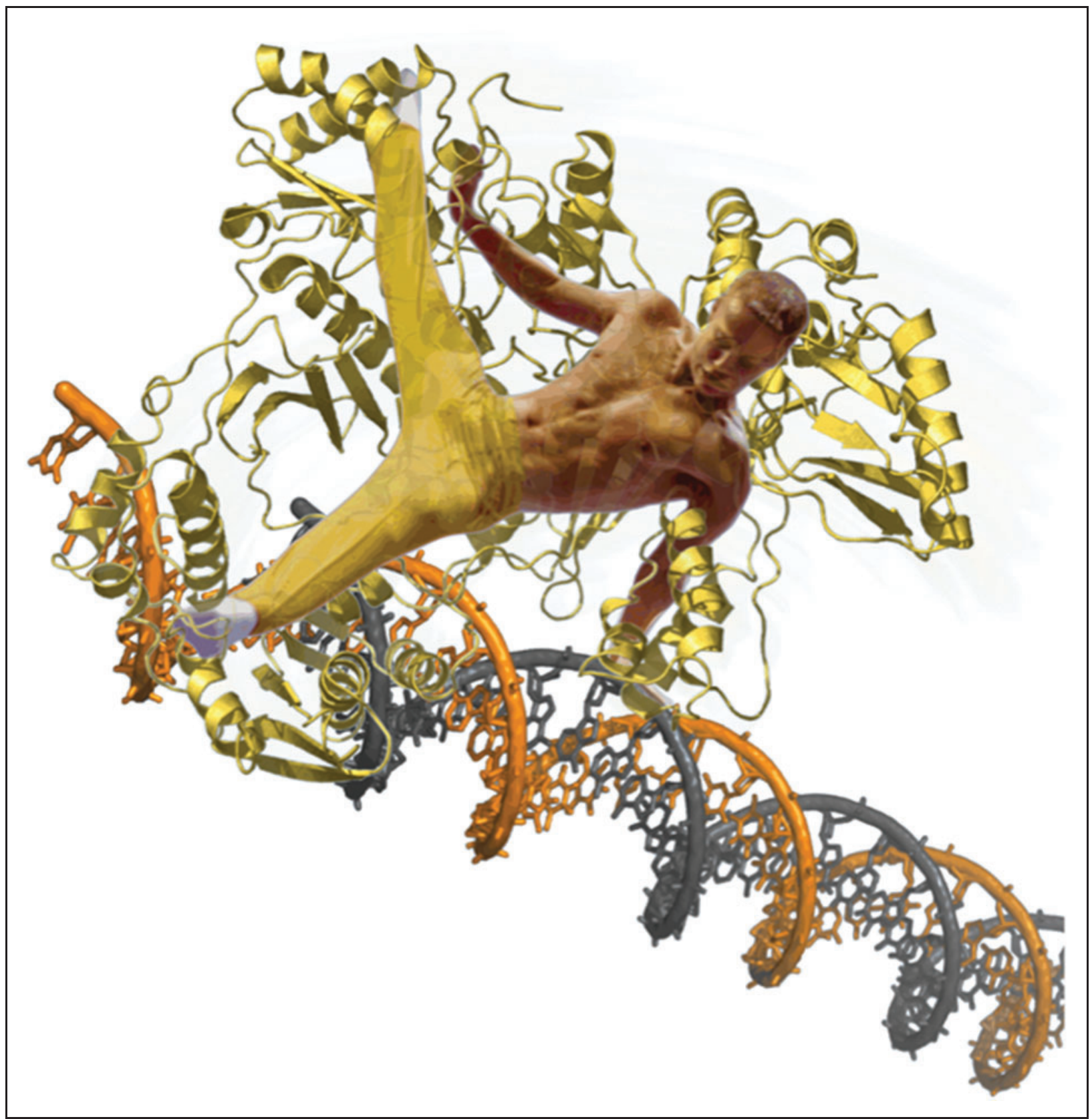

FIG. 1. Multistep acrobatics and reverse transcription.

${ }^{1}$ Department of Bionanoscience, Delft University of Technology, Delft, The Netherlands.

${ }^{2}$ RT Biochemistry Section, HIV Drug Resistance Program, National Cancer Institute-Frederick, Frederick, Maryland. 
YNTHesizing InTEGRATION-COMPETENT, double-stranded DNA from the single-stranded human immunodeficiency virus (HIV) RNA genome might more aptly be described as molecular gymnastics (Fig. 1). Key elements of this routine include intramolecular and intermolecular transfer of the replication complex between genome termini, strand displacement synthesis, selecting plus-strand RNA primers from within the RNA/DNA replication intermediate, their excision from nascent DNA, and phased A-tract-induced cessation of DNA synthesis near the center of the genome. While sitedirected mutagenesis identifies residues of HIV-1 reverse transcriptase (RT) that mediate these functions, and X-ray crystallography gives elegant, but static snapshots of nucleoprotein complexes, they cannot assess conformational dynamics of this remarkably versatile enzyme. To better understand how HIV-1 RT interacts with nucleic acid required designing a single molecule strategy based on Foerster resonance energy transfer (FRET), placing fluorophores into the enzyme and its nucleic acid substrates.

Studies with duplex DNA predictably recapitulated an enzyme with its DNA polymerase catalytic center straddled over the primer terminus. However, an unexpected observation of Abbondanzieri et al. ${ }^{1}$ was that this orientation reversed when the DNA primer was replaced with RNA. Later studies examining polypurine tract recognition not only confirmed that HIV-1 RT could assume different orientations, but also was sufficiently agile to "flip" between these during a single binding event. Such reversal in RT orientation could help explain how its N-terminal DNA polymerase and C-terminal ribonuclease $\mathrm{H}$ (RNase $\mathrm{H})$ domains can recognize the PPT primer terminus at different steps of plus-strand synthesis.

Another concept we accept when studying HIV-1 RT is that it is a distributive polymerase, i.e., it frequently dissociates from its nucleic acid substrate. How then, upon rebinding, does the enzyme locate the primer terminus to reengage in DNA synthesis? Subsequent studies of Liu et al. illustrated the capacity of RT to slide on the nucleic acid duplex, a property that could be enhanced in the presence of nonnucleoside RT inhibitors or restricted by forming a ternary complex. However, the story does not end here. Since there appears to be no orientation (or directional) bias during sliding, the polymerization target site could be accessed by the retroviral polymerase in two different orientations. Single molecule spectroscopy not only confirmed this, but elegantly demonstrated that an enzyme accessing the primer terminus in an "RNase H mode" could spontaneously flip into the polymerization mode, thereby linking the properties of flipping and sliding. ${ }^{2}$

A state-of-the-art technology, high-profile publications, but what is the significance? For many years, we have presumed that nonnucleoside inhibition of HIV-1 RT occurred through a "grinding of the gears" and an inability to support DNA synthesis. However, examining the interaction of HIV-1 RT with its PPT by single molecule spectroscopy revealed that the nonnucleoside reverse transcriptase inhibitor (NNRTI) nevirapine promotes a reversal of enzyme orientation, positioning the RNase $\mathrm{H}$ domain over the primer terminus, thereby inhibiting synthesis of plus-strand DNA. These and related studies on tRNA-primed initiation of minus-strand DNA synthesis ${ }^{3}$ show that, in a different sense, HIV fitness requires nimble and flexible maneuvering of its DNA polymerase!

\section{References}

1. Abbondanzieri EA, Bokinsky G, Rausch JW, Zhang J, Le Grice SFJ, and Zhuang X: Dynamic binding orientations determine the enzymatic activity of HIV reverse transcriptase. Nature 2008;453:184-189.

2. Liu S, Abbondanzieri EA, Rausch JW, Le Grice SFJ, and Zhuang X: Slide into action dynamic shuttling of HIV reverse transcriptase on nucleic acid substrates. Science 2008;322:1092-1097.

3. Liu S, Harada BT, Miller JT, Le Grice SFJ, and Zhuang X: Initiation complex dynamics direct the transitions between distinct phases of early HIV reverse transcription. Nat Struct Mol Biol 2010;17:1453-1460.

Address correspondence to: Stuart F.J. Le Grice RT Biochemistry Section HIV Drug Resistance Program National Cancer Institute-Frederick Frederick, Maryland 21702

E-mail: legrices@mail.nih.gov 